\title{
A Simple Proof of the Generalized Riemann's Hypothesis
}

\author{
Charaf ECH-CHATBI *
}

1 September 2022

\begin{abstract}
This article is the continuation of the previous $\mathrm{RH}$ proof 1 . We present a simple proof of the Riemann's Hypothesis for Dirichlet $L$-functions where we apply the same method we used to prove $\mathrm{RH}$.
\end{abstract}

Keywords: Riemann Hypothesis; Zeta function; Prime Numbers; Millennium Problems.

MSC2020 Classification: 11Mxx, 11-XX, 26-XX, 30-xx.

\section{Dirichlet $L$-functions}

Let's $\left(z_{n}\right)_{n \geq 1}$ be a sequence of complex numbers. A Dirichlet series[3] is a series of the form $\sum_{n=1}^{\infty} \frac{z_{n}}{n^{s}}$, where $s=\sigma+i \tau$ is complex. The zeta function is a Dirichlet series. Let's define the function $L(s)$ of the complex $s$ : $L(s)=\sum_{n=1}^{\infty} \frac{z_{n}}{n^{s}}$.

- If $\left(z_{n}\right)_{n \geq 1}$ is a bounded, then the corresponding Dirichlet series converges absolutely on the open half-plane where $\Re(s)>1$.

- If the set of sums $z_{n}+z_{n+1}+\ldots+z_{n+k}$ for each $n$ and $k \geq 0$ is bounded, then the corresponding Dirichlet series converges on the open half-plane where $\Re(s)>0$.

- In general, if $z_{n}=O\left(n^{k}\right)$, the corresponding Dirichlet series converges absolutely in the half plane where $\Re(s)>k+1$.

The function $L(s)$ is analytic on the corresponding open half plane[2-3].

To define Dirichlet $L$-functions we need to define Dirichlet characters. A function $\chi: \mathbb{Z} \longmapsto \mathbb{C}$ is a Dirichlet character modulo $q$ if it satisfies the following criteria:

*One Raffles Quay, North Tower. 048583 Singapore. Email: charaf.chatbi@gmail.com. The opinions of this article are those of the author and do not reflect in any way the views or business of his employer. 
- (i) $\chi(n) \neq 0$ if $(n, q)=1$.

- (ii) $\chi(n)=0$ if $(n, q)>1$.

- (iii) $\chi$ is periodic with period $q: \chi(n+q)=\chi(n)$ for all $\mathrm{n}$.

- (iv) $\chi$ is multiplicative $: \chi(m n)=\chi(m) \chi(n)$ for all integers $m$ and $\mathrm{n}$.

The trivial character is the one with $\chi_{0}(n)=1$ whenever $(n, q)=1$.

Here are some known results for a Dirichlet character modulo $q$. For any integer $n$ we have $\chi(1)=1$. Also if $(n, q)=1$, we have $(\chi(n))^{\phi(q)}=$ 1 with $\phi$ is Euler's totient function. $\chi(n)$ is a $\phi(q)$-th root of unity. Therefore, $|\chi(n)|=1$ if $(n, q)=1$, and $|\chi(n)|=0$ if $(n, q)>1$.

Also, we recall the cancellation property for Dirichlet characters modulo $q$ : For any $n$ integer

$$
\sum_{i=1}^{q} \chi(i+n)= \begin{cases}\phi(q), & \text { if } \chi=\chi_{0} \text { the trivial character } \\ 0, & \text { if otherwise, } \chi \neq \chi_{0}\end{cases}
$$

The Dirichlet $L$-functions are simply the sum of the Dirichlet series. Let's $\chi$ be a Dirichlet character modulo $q$, The Dirichlet $L$-function $L(s, \chi)$ is defined for $\Re(s)>1$ as the following:

$$
L(s, \chi)=\sum_{n=1}^{+\infty} \frac{\chi(n)}{n^{s}}
$$

They are a natural generalization of the Riemann zeta-function $\zeta(s)$ to an arithmetic progression and are a powerful tool in analytic number theory. The Dirichlet series, converges absolutely and uniformly in any bounded domain in the complex $s$-plane for which $\Re(s) \geq 1+\gamma, \gamma>0$. In the particular case of the trivial character $\chi_{0}, L\left(s, \chi_{0}\right)$ extends to a meromorphic function[2-5] in $\Re(s)>0$ with the only pole at $s=1$.

If $\chi$ is a non-trivial character, we have

$$
L(s, \chi)=s \int_{1}^{+\infty} \frac{\sum_{n=1}^{n \leq x} \chi(n)}{x^{s+1}} d x
$$

Since the sum in the integrand is bounded, this formula gives an analytic continuation of $L(s, \chi)$ to a regular function in the half-plane $\Re(s)>0$. Also, like the zeta function, the Dirichlet $L$-functions have their Euler product[1-2]. For $\Re(s)>1$ :

$$
L(s, \chi)=\prod_{p \text { Prime }}\left(1-\frac{\chi(p)}{p^{s}}\right)^{-1}
$$

Therefore, if $\chi=\chi_{0}$ is a trivial character $\bmod q$, we have, for $q=1$,

$$
L\left(s, \chi_{0}\right)=\zeta(s)
$$

And for $q>1$, we have,

$$
L\left(s, \chi_{0}\right)=\zeta(s) \prod_{p / q}\left(1-\frac{1}{p^{s}}\right)
$$


For this reason the properties of $L\left(s, \chi_{0}\right)$ in the entire complex plane are determined by the properties of $\zeta(s)$.

Let's now $q^{\prime}$ be the smallest divisor (prime) of $q$. Let's $\chi^{\prime}$ be the Dirichlet character $\chi^{\prime} \bmod q \prime$. For any integer $n$ such that $(n, q)=1$, we have also $\left(n, q^{\prime}\right)=1$ and $\chi(n)=\chi^{\prime}(n) . \chi^{\prime}$ is called primitive and $L(s, \chi)$ and $L\left(s, \chi^{\prime}\right)$ are related analytically such that if $\chi \neq \chi_{0}$ :

$$
L(s, \chi)=L\left(s, \chi^{\prime}\right) \prod_{p / q}\left(1-\frac{\chi^{\prime}(p)}{p^{s}}\right)
$$

$L(s, \chi)$ and $L\left(s, \chi^{\prime}\right)$ have the same zeros in the critical strip $0<\Re(s)<1$.

Also, for a primitive character $\chi,\left(\right.$ i.e. $\left.\chi=\chi^{\prime}\right) L(s, \chi)$ has the following functional equation:

$$
\tau(\chi) \Gamma\left(\frac{1-s+a}{2}\right) L(1-s, \chi)=\sqrt{\pi}\left(\frac{q}{\pi}\right)^{s} i^{a} q^{\frac{1}{2}} \Gamma\left(\frac{s+a}{2}\right) L(s, \bar{\chi})
$$

Where $\Gamma$ is the Gamma function and $a=0$ if $\chi(-1)=1$ and $a=1$ if $\chi(-1)=-1$, and $\tau(\chi)=\sum_{k=1}^{q} \chi(k) \exp \left(\frac{2 \pi k i}{q}\right)$.

When $\Re(s)>1$ there is no zero for $L(s, \chi)$. When $\Re(s)<0$, for a primitive character $\chi$, we have the trivial zeros of $L(s, \chi): s=a-2 k$, where $k$ is a positive integer and $a$ is defined above. For more details, please refer to the references[1-8].

\section{The Generalized Riemann Hypothesis}

The Generalized Riemann Hypothesis states that the Dirichlet $L$-functions have all their non-trivial zeros on the critical line $\Re(s)=\frac{1}{2}$.

We know that for any character $\chi$ modulo $q$, all non-trivial zeros of $L(s, \chi)$ lies in the critical strip $\{s \in \mathbb{C}: 0<\Re(s)<1\}$. From the functional equation above we have that if:

- $s_{0}$ is a non-trivial zero of $L(s, \chi)$, then $1-s_{0}$ is a zero of $L(s, \bar{\chi})$.

- $s_{0}$ is a non-trivial zero of $L(s, \bar{\chi})$, then $1-s_{0}$ is a zero of $L(s, \chi)$.

Therefore, we just need to prove that for any character 1$]$ modulo $q$, there is no non-trivial zeros of $L(s, \chi)$ in the right hand side of the critical strip $\left\{s \in \mathbb{C}: \frac{1}{2}<\Re(s)<1\right\}$.

\section{Proof of the Generalized Riemann Hy- pothesis}

Let's take a complex number $s$ such that $s=\sigma+i \tau$. Unless we explicitly mention otherwise, let's suppose that $0<\sigma<1, \tau>0$ and $L(s, \chi)=0$ where $L(s, \chi)=\sum_{n=1}^{+\infty} \frac{\chi(n)}{n^{s}}$ and $\chi$ is a non-trivial Dirichlet character $\chi$ modulo $q$. When $q=2$ there is only one Dirichlet character and it is

\footnotetext{
${ }^{1}$ In fact, we just need to prove it for the primitive characters.
} 
trivial. So here we are going to assume that $q \geq 3$.

We have from the integral equation (3) above:

$$
L(s, \chi)=s \int_{1}^{+\infty} \frac{\sum_{n=1}^{n \leq x} \chi(n)}{x^{s+1}} d x
$$

We have from the Riemann's integral in [1]:

$$
\zeta(s)=\frac{s}{s-1}-s \int_{1}^{+\infty} \frac{\{x\}}{x^{s+1}} d x
$$

In this article, we leverage on the $\mathrm{RH}$ proof in [1] to proof GRH. Also, we will use the same method and notations as in [1]. Let's recall from [1] the following notations:

$$
\begin{aligned}
\epsilon_{0}(x) & =\{x\} \\
\epsilon_{n+1}(x) & =\frac{1}{x} \int_{0}^{x} d t \epsilon_{n}(t) \\
\epsilon(x) & =\sum_{k=0}^{+\infty} \epsilon_{k}(x)
\end{aligned}
$$

Lemma 3.1. Let's consider two variables $\sigma$ and $\tau$ such that $\sigma>0, \tau>0$ and $s=\sigma+i \tau$. Let's $L(s, \chi)$ be a L-function where $\chi$ is a character (not necessarily a Dirichlet character) such that:

$$
L(s, \chi)=\frac{\lambda s}{s-1}-s \int_{1}^{+\infty} \frac{\left(\lambda\{x\}+f_{\lambda}(x)\right)}{x^{s+1}} d x
$$

With the function $x \rightarrow f_{\lambda}(x)$ is bounded over $\mathbb{R}^{+}$and null over the interval $[0,1]$ and the complex constant $\lambda \neq 0$. Let's define the sequence of functions $f_{n}$ and $\bar{f}_{n}$ such that $f_{0}(x)=\bar{f}_{0}(x)=\frac{f_{\lambda}(x)}{\lambda}$ and for each $n \geq 1$ :

$$
\begin{aligned}
f_{n+1}(x) & =\frac{1}{x} \int_{0}^{x} d t f_{n}(t) \\
\bar{f}_{n+1}(x) & =\frac{1}{x} \int_{1}^{x} d t \bar{f}_{n}(t) \\
f(x) & =\sum_{k=0}^{+\infty} f_{k}(x)
\end{aligned}
$$

If $L(s, \chi)=0$, then:

1. For each $n$ :

$$
\int_{1}^{+\infty} d x \frac{\epsilon_{n}(x)+f_{n}(x)}{x^{1+s}}=-\frac{1}{(1-s) 2^{n}}
$$

2. For each $n$ :

$$
\int_{1}^{+\infty} d x \frac{\epsilon_{n}(x)+f_{n}(x)}{x^{2-s}}=-\frac{1}{(s) 2^{n}}
$$


3. For each $x \geq 1$ :

$$
f_{n}(x)=\bar{f}_{n}(x)
$$

4. For each $n$ and $x \geq 1$ :

$$
f_{n}(x)=\frac{1}{(n-1) !} \frac{1}{x} \int_{1}^{x} d t f_{0}(t)\left(\ln \left(\frac{x}{t}\right)\right)^{n-1}
$$

5. For each $x \geq 1$ :

$$
\lim _{n \rightarrow+\infty} 2^{n} f_{n}(x)=0
$$

6. For each $x \geq 0$ :

$$
f(x)=f_{0}(x)+\int_{1}^{x} d t \frac{f_{0}(t)}{t}
$$

7. There exists a constant $M>0$ such that for each $x \geq 0$ :

$$
|\epsilon(x)+f(x)| \leq 2+M+(M+1) \ln (x)
$$

Proof. Let's take $s$ such that $L(s, \chi)=0$, therefore we can write:

$$
\frac{\lambda s}{s-1}-s \int_{1}^{+\infty} \frac{(\lambda\{x\}+f(x))}{x^{s+1}} d x=0
$$

Therefore, since $s \neq 0$ and $\lambda \neq 0$, we have:

$$
\int_{1}^{+\infty} \frac{\epsilon_{0}(x)+f_{0}(x)}{x^{s+1}} d x=\frac{1}{s-1}
$$

We use the same method used in the lemma 1.2 in [1] to prove this lemma.

Lemma 3.2. Let's consider two variables $\sigma$ and $\tau$ such that $0<\sigma \leq \frac{1}{2}$, $\tau>0$ and $s=\sigma+i \tau$. Let's $L(s, \chi)$ be a $L$-function where $\chi$ is a character (not necessarily a Dirichlet character) such that:

$$
L(s, \chi)=\frac{\lambda s}{s-1}-s \int_{1}^{+\infty} \frac{(\lambda\{x\}+f(x))}{x^{s+1}} d x
$$

With the function $x \rightarrow f(x)$ is bounded over $\mathbb{R}^{+}$and null over the interval $[0,1]$ and the complex constant $\lambda \neq 0$.

Let's define the sequence of functions $E_{n, \sigma}(x), F_{n, \sigma}(x), G_{n, \sigma}(x)$ and $H_{n, \sigma}(x)$ over $[1,+\infty)$ for each $n \geq 1$ as follows:

$$
\begin{aligned}
& E_{n, \sigma}(x)=\Im\left[\int_{1}^{x} d t \frac{\left(f_{n}(t)+\epsilon_{n}(t)\right)}{t^{1+s}}\right] \\
& F_{n, \sigma}(x)=\Re\left[\int_{1}^{x} d t \frac{\left(f_{n}(t)+\epsilon_{n}(t)\right)}{t^{1+s}}\right] \\
& G_{n, \sigma}(x)=\left(E_{n, \sigma}(x)\right)^{2}+\left(F_{n, \sigma}(x)\right)^{2} \\
& H_{n, \sigma}(x)=G_{n, 1-\sigma}(x)-G_{n, \sigma}(x)
\end{aligned}
$$


Where $\Im(z)$ and $\Re(z)$ are the imaginary and the real parts of the complex $z$. Let's denote $a_{0}=e^{\frac{3}{2 \tau}}$ and $b_{0}=e^{\frac{\pi}{2 \tau}}$.

If $L(s, \chi)=0$, then:

1. There is exist a large enough integer $N_{0}$ such that for each $n \geq N_{0}$, there exists $x_{n}>b_{0}$ such as:

$$
H_{n, \sigma}\left(x_{n}\right)=0
$$

2. There is exist a large enough integer $N_{0}$ such that for each $n \geq N_{0}$, there exists $x_{n}>b_{0}$ such as:

$$
\sum_{k=n+1}^{+\infty} H_{k, \sigma}\left(x_{n}\right)=0
$$

In other terms:

$$
\sum_{k=n+1}^{+\infty} G_{k, 1-\sigma}\left(x_{n}\right)=\sum_{k=n+1}^{+\infty} G_{k, \sigma}\left(x_{n}\right)
$$

Proof. Let's prove the first point. We have $\sigma \leq \frac{1}{2}$ and $a_{0}=e^{\frac{3}{2 \tau}}, b_{0}=e^{\frac{\pi}{2 \tau}}$. From lemma 1.2 in [1, we have:

$$
\lim _{n \rightarrow+\infty} 2^{n} \epsilon_{n}(x)=x
$$

And from lemma 3.1, we have:

$$
\lim _{n \rightarrow+\infty} 2^{n} f_{n}(x)=0
$$

Therefore for each $x \geq 1$ :

$$
\begin{aligned}
& \lim _{n \rightarrow+\infty} \Im\left[\frac{2^{n}\left(\epsilon_{n}(x)+f_{n}(x)\right)}{x^{1+s}}\right]=-\frac{\sin (\tau \ln (x))}{x^{\sigma}} \\
& \lim _{n \rightarrow+\infty} \Re\left[\frac{2^{n}\left(\epsilon_{n}(x)+f_{n}(x)\right)}{x^{1+s}}\right]=\frac{\cos (\tau \ln (x))}{x^{\sigma}} \\
& \lim _{n \rightarrow+\infty} \Im\left[\frac{2^{n}\left(\epsilon_{n}(x)+f_{n}(x)\right)}{x^{2-s}}\right]=\frac{\sin (\tau \ln (x))}{x^{1-\sigma}} \\
& \lim _{n \rightarrow+\infty} \Re\left[\frac{2^{n}\left(\epsilon_{n}(x)+f_{n}(x)\right)}{x^{2-s}}\right]=\frac{\cos (\tau \ln (x))}{x^{1-\sigma}}
\end{aligned}
$$

Thanks to the Dominated Convergence Theorem applied on the sequence of functions in $(37-40)$ over the bounded interval $\left[1, a_{0}\right]$, we can write:

$$
\begin{aligned}
\lim _{n \rightarrow+\infty} 2^{2 n} G_{n, \sigma}\left(a_{0}\right) & =\left(\int_{1}^{a_{0}} d t \frac{\sin (\tau \ln (t))}{t^{\sigma}}\right)^{2}+\left(\int_{1}^{a_{0}} d t \frac{\cos (\tau \ln (t))}{t^{\sigma}}\right)^{2} \\
\lim _{n \rightarrow+\infty} 2^{2 n} G_{n, 1-\sigma}\left(a_{0}\right) & =\left(\int_{1}^{a_{0}} d t \frac{\sin (\tau \ln (t))}{t^{1-\sigma}}\right)^{2}+\left(\int_{1}^{a_{0}} d t \frac{\cos (\tau \ln (t))}{t^{1-\sigma}}\right)^{2}
\end{aligned}
$$


Thanks to lemma 1.4 in [1, we can calculate the integrals in the equations $(41-42)$, we conclude:

$$
\begin{array}{rll}
2^{2 n} G_{n, \sigma}\left(a_{0}\right) & \sim_{n \rightarrow+\infty} & f_{a_{0}}(1-\sigma)=\frac{a_{0}^{2(1-\sigma)}-2 a_{0}^{(1-\sigma)} \cos \left(\tau \ln \left(a_{0}\right)\right)+1}{(1-\sigma)^{2}+\tau^{2}} \\
2^{2 n} G_{n, 1-\sigma}\left(a_{0}\right) & \sim_{n \rightarrow+\infty} & f_{a_{0}}(\sigma)=\frac{a_{0}^{2 \sigma}-2 a_{0}^{\sigma} \cos \left(\tau \ln \left(a_{0}\right)\right)+1}{\sigma^{2}+\tau^{2}}
\end{array}
$$

Where $f_{a}$ is strictly increasing as we saw in lemma 1.4 in [1]. Therefore

$$
\lim _{n \rightarrow+\infty} 2^{2 n} H_{n, \sigma}\left(a_{0}\right)=f_{a_{0}}(\sigma)-f_{a_{0}}(1-\sigma)<0
$$

Therefore, there is exist a large enough integer $N_{0}$ such that for each $n \geq N_{0}$ :

$$
2^{2 n} H_{n, \sigma}\left(a_{0}\right)<0
$$

Let's take now $n \geq N_{0}$. From lemma 3.1, have:

$$
\begin{array}{r}
2^{n} \int_{1}^{+\infty} d t \frac{\left(\epsilon_{n}(t)+f_{n}(t)\right)}{t^{1+s}}=-\frac{1}{(1-s)} \\
2^{n} \int_{1}^{+\infty} d t \frac{\left(\epsilon_{n}(t)+f_{n}(t)\right)}{t^{2-s}}=-\frac{1}{(s)}
\end{array}
$$

Therefore:

$$
\begin{aligned}
\lim _{x \rightarrow+\infty} G_{n, \sigma}(x) & =\Im\left(\int_{1}^{+\infty} d t \frac{\left(\epsilon_{n}(t)+f_{n}(t)\right)}{t^{1+s}}\right)^{2}+\Re\left(\int_{1}^{+\infty} d t \frac{\left(\epsilon_{n}(t)+f_{n}(t)\right)}{t^{1+s}}\right)^{2} \\
& =\frac{1}{2^{2 n}\|1-s\|^{2}}
\end{aligned}
$$

And the same for the case with $1-s$, we can write:

$$
\begin{aligned}
\lim _{x \rightarrow+\infty} G_{n, 1-\sigma}(x) & =\Im\left(\int_{1}^{+\infty} d t \frac{\left(\epsilon_{n}(t)+f_{n}(t)\right)}{t^{2-s}}\right)^{2}+\Re\left(\int_{1}^{+\infty} d t \frac{\left(\epsilon_{n}(t)+f_{n}(t)\right)}{t^{2-s}}\right)^{2} \\
& =\frac{1}{2^{2 n}\|s\|^{2}}
\end{aligned}
$$

Therefore:

$$
\lim _{x \rightarrow+\infty} H_{n, \sigma}(x)=\frac{1}{2^{2 n}}\left(\frac{1}{\|s\|^{2}}-\frac{1}{\|1-s\|^{2}}\right)>0
$$

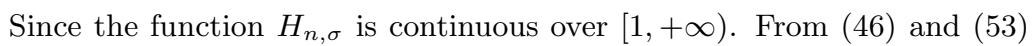
and thanks to the Mean value theorem, we can conclude that there exists an $x_{n}>b_{0}$ such that:

$$
H_{n, \sigma}\left(x_{n}\right)=0
$$


To prove the second point, we use the same method as in the second point of lemma 1.3 in [1]. Let's $N_{0}$ be the same as defined during the first point. Let's define now the sequence of functions $s(n, \sigma, x)$ such that for $n \geq N_{0}$ and $x \geq 1$ :

$$
s(n, \sigma, x)=\sum_{k=N_{0}}^{n} G_{k, \sigma}(x)
$$

From the equations (45-46), we can deduce that:

$$
\sum_{k=N_{0}}^{+\infty} H_{k, \sigma}\left(a_{0}\right) \leq 0
$$

In order to use the Mean Value theorem like in the point 1) of this lemma, 1) we will prove that the function $s(+\infty, \sigma, x)(\operatorname{resp} . s(+\infty, 1-$ $\sigma, x))$ is continuous over $[1,+\infty)$. and 2$)$ we will prove that:

$$
\lim _{x \rightarrow+\infty} \sum_{k=N_{0}}^{+\infty} H_{k, \sigma}(x)=\frac{1}{34^{N_{0}-1}}\left(\frac{1}{\|s\|^{2}}-\frac{1}{\|1-s\|^{2}}\right)>0
$$

Let's take a sequence $\left(y_{n} \geq 1\right)$ such that it has a limit $y . y$ can be finite or $+\infty$. The proof is similar for both cases. So, let's consider the double sequence $s(n, \sigma, m)$ such that:

$$
s(n, \sigma, m)=s\left(n, \sigma, y_{m}\right)
$$

We will prove that the double sequence $(s(n, \sigma, m))$ is a Cauchy sequence using the same method as in lemma 1.3 in [1]. We will use the points (6) and (7) of the lemma 3.1 to support the same steps as in [1].

Lemma 3.3. Let's consider two variables $\sigma$ and $\tau$ such that $0<\sigma \leq \frac{1}{2}$, $\tau>0$ and $s=\sigma+i \tau$. Let's $L(s, \chi)$ be a $L$-function where $\chi$ is a character (not necessarily a Dirichlet character) such that:

$$
L(s, \chi)=\frac{\lambda s}{s-1}-s \int_{1}^{+\infty} \frac{(\lambda\{x\}+f(x))}{x^{s+1}} d x
$$

With the function $x \rightarrow f(x)$ is bounded over $\mathbb{R}^{+}$and null over the interval $[0,1]$ and the complex constant $\lambda \neq 0$.

If $L(s, \chi)=0$, then:

$$
\sigma=\frac{1}{2}
$$

Proof. With the result of lemmas 3.1 and 3.2, we use the same methods used in lemmas $1.4,1.5$ and 1.6 in [1] to prove this lemma.

From the lemma 3.3 above we can conclude the following corollary. 
Corollary 3.3.1. Let's consider two variables $\sigma$ and $\tau$ such that $\sigma>0$, $\tau>0$ and $s=\sigma+i \tau$. Let's $q>1$ be an positive integer. Let's $L(s, \chi)$ be a L-function where $\chi$ is a Dirichlet character mod $q$ with no cancellation property such that:

$$
\sum_{n=1}^{q} \chi(n) \neq 0
$$

If $L(s, \chi)=0$, then:

$$
\sigma=\frac{1}{2}
$$

Proof. Let's take $x>1$ a real number. Let's denote $n_{x}=\lfloor x\rfloor$ be the integer part of $x$. We have $n_{x}=x-\{x\}$. We denote $q_{x}$ and $r_{x}$ the quotient and the rest of the Euclidean division of $n_{x}$ by $q$ as follows: $n_{x}=q q_{x}+r_{x}$. Therefore we can write:

$$
\begin{aligned}
\sum_{n=1}^{x} \chi(n) & =\sum_{n=0}^{q_{x}-1} \sum_{k=1}^{q} \chi(n q+k)+\sum_{k=1}^{r_{x}} \chi(n q+k) \\
& =q_{x} \sum_{n=1}^{q} \chi(n)+\sum_{k=1}^{r_{x}} \chi(k) \\
& =\frac{x-\{x\}-r_{x}}{q} \sum_{k=1}^{q} \chi(n)+\sum_{k=1}^{r_{x}} \chi(k) \\
& =\frac{x}{q} \sum_{k=1}^{q} \chi(n)-\frac{\left(\{x\}+r_{x}\right)}{q} \sum_{k=1}^{q} \chi(n)+\sum_{k=1}^{r_{x}} \chi(k)
\end{aligned}
$$

Let's define the constant $\lambda$ and the function $g$ as follows:

$$
\begin{aligned}
\lambda & =\frac{\sum_{k=1}^{q} \chi(n)}{q} \\
g(x) & =\frac{\left(\{x\}+r_{x}\right)}{q} \sum_{k=1}^{q} \chi(n)-\sum_{k=1}^{r_{x}} \chi(k) \\
& =\lambda\{x\}+\underbrace{\lambda r_{x}-\sum_{k=1}^{r_{x}} \chi(k)}_{f(x)}
\end{aligned}
$$

It is clear that the function $f(x)$ is bounded over $\mathbb{R}^{+}$and null over $[0,1]$. And by assumption we have $\lambda \neq 0$. Therefore, we can calculate $L(s, \chi)$ as follows:

$$
\begin{aligned}
L(s, \chi) & =s \int_{1}^{+\infty} \frac{\sum_{n=1}^{n \leq x} \chi(n)}{x^{s+1}} d x \\
& =s \int_{1}^{+\infty} \frac{\lambda x-g(x)}{x^{s+1}} d x \\
& =s \int_{1}^{+\infty} \frac{\lambda}{x^{s}} d x-s \int_{1}^{+\infty} \frac{\lambda\{x\}+f(x)}{x^{s+1}} d x
\end{aligned}
$$


If $\Re(s)>1$, we can conclude:

$$
L(s, \chi)=\frac{\lambda s}{s-1}-s \int_{1}^{+\infty} \frac{\lambda\{x\}+f(x)}{x^{s+1}} d x
$$

We can extend the equation (74) over the region $\Re(s)>0 . L(s, \chi)$ has a holomorphic continuation as a function of $s$ to the whole complex plane (see $[3,6,7,8]$ ). Therefore, we apply the lemma 3.3 to prove that $\sigma=\frac{1}{2}$.

Remark. As a direct result of the above corollary, we can see that GRH is true for the series $\left(\sum_{n \geq 1} \frac{1}{(q n+p)^{s}}\right)$ with $p>0$ and $q>1$ are integers.

Lemma 3.4. Let's consider two variables $\sigma$ and $\tau$ such that $\sigma>0, \tau>0$ and $s=\sigma+i \tau$. Let's $q>1$ be an positive integer. Let's $L(s, \chi)$ be a L-function where $\chi$ is a Dirichlet character mod $q$ with cancellation property such that:

$$
\sum_{n=1}^{q} \chi(n)=0
$$

Therefore:

$$
L(s, \chi)=\left(\frac{1-q^{1-s}}{q}\right) \frac{s}{s-1}-s \int_{1}^{+\infty} \frac{\left(\frac{1-q^{1-s}}{q}\right)\{x\}+f(x)}{x^{s+1}} d x
$$

Where $f$ is a bounded function over $\mathbb{R}^{+}$and null over the interval $[0,1]$.

Proof. We will use the zeta function $\zeta(s)$ to prove our case.

Let's now write the following for $s>1$ :

$$
\begin{aligned}
\zeta(s) & =\sum_{n=1}^{+\infty} \frac{1}{n^{s}} \\
& =\sum_{n=0}^{\infty} \sum_{i=1}^{q} \frac{1}{(q n+i)^{s}} \\
& =\sum_{i=1}^{q} \sum_{n=0}^{\infty} \frac{1}{(q n+i)^{s}}
\end{aligned}
$$

Therefore

$$
\left(1-\frac{1}{q^{s}}\right) \zeta(s)=\sum_{i=1}^{q-1} \sum_{n=0}^{\infty} \frac{1}{(q n+i)^{s}}
$$

Also

$$
\begin{aligned}
L(s, \chi) & =\sum_{n=1}^{+\infty} \frac{\chi(n)}{n^{s}} \\
& =\sum_{n=0}^{+\infty} \sum_{i=1}^{q} \frac{\chi(i)}{(q n+i)^{s}} \\
& =\sum_{i=1}^{q} \chi(i) \sum_{n=0}^{+\infty} \frac{1}{(q n+i)^{s}}
\end{aligned}
$$


Therefore since $\chi(q)=0$

$$
L(s, \chi)=\sum_{i=1}^{q-1} \chi(i) \sum_{n=0}^{+\infty} \frac{1}{(q n+i)^{s}}
$$

Since $\sum_{n=1}^{q} \chi(n)=0$ and $\chi(1)=1$, we can write the following:

$$
\begin{aligned}
& \sum_{n=0}^{+\infty} \frac{1}{(q n+1)^{s}}=L(s, \chi)-\sum_{i=2}^{q-1} \chi(i) \sum_{n=0}^{+\infty} \frac{1}{(q n+i)^{s}} \\
& \sum_{n=0}^{+\infty} \frac{1}{(q n+1)^{s}}=\left(1-\frac{1}{q^{s}}\right) \zeta(s)-\sum_{i=2}^{q-1} \sum_{n=0}^{+\infty} \frac{1}{(q n+i)^{s}}
\end{aligned}
$$

Therefore

$$
\left(1-\frac{1}{q^{s}}\right) \zeta(s)-L(s, \chi)=\sum_{i=2}^{q-1}(1-\chi(i)) \sum_{n=0}^{+\infty} \frac{1}{(q n+i)^{s}}
$$

Let's denote $L(s, i)_{2 \leq i \leq q-1}$ as follows:

$$
L(s, i)=\sum_{n=0}^{+\infty} \frac{1}{(q n+i)^{s}}
$$

We can write also:

$$
L(s, i)=s \int_{1}^{+\infty} \frac{\sum_{n=1}^{n \leq x} \chi_{i}(n)}{x^{s+1}} d x
$$

Where $\chi_{i}$ is the character of $L(s, i)$ :

- (i) $\chi_{i}(q n+i)=1$

- (ii) $\chi_{i}(q n+j)=0$ if $j \neq i$

Let's take $x>1$ a real number. Let's denote $n_{x}=\lfloor x\rfloor$ be the integer part of $x$. We have $n_{x}=x-\{x\}$. We denote $q_{x}$ and $r_{x}$ the quotient and the rest of the Euclidean division of $n_{x}$ by $q$ as follows: $n_{x}=q q_{x}+r_{x}$. Let's denote the following:

$$
\begin{aligned}
\lambda_{i} & =\frac{\sum_{k=1}^{q} \chi_{i}(n)}{q}=\frac{1}{q} \\
f_{i}(x) & =\frac{\left(\{x\}+r_{x}\right)}{q} \sum_{k=1}^{q} \chi_{i}(n)-\sum_{k=1}^{r_{x}} \chi_{i}(k) \\
& =\frac{\left(\{x\}+r_{x}\right)}{q}-\sum_{k=1}^{r_{x}} \chi_{i}(k)
\end{aligned}
$$

Therefore from the equation (74):

$$
L(s, i)=\frac{s}{q(s-1)}-s \int_{1}^{+\infty} \frac{f_{i}(x)}{x^{s+1}} d x
$$


Therefore, we can write the equation (87) as follows:

$$
\begin{aligned}
\left(1-\frac{1}{q^{s}}\right) \zeta(s)-L(s, \chi) & =\sum_{i=2}^{q-1}(1-\chi(i)) L(s, i) \\
& =\frac{s}{q(s-1)} \sum_{i=2}^{q-1}(1-\chi(i))-s \sum_{i=2}^{q-1}(1-\chi(i)) \int_{1}^{+\infty} \frac{f_{i}(x)}{x^{s+1}} d x
\end{aligned}
$$

Thanks to the cancellation property, we can conclude that:

$$
\left(1-\frac{1}{q^{s}}\right) \zeta(s)-L(s, \chi)=\frac{s(q-1)}{q(s-1)}-s \int_{1}^{+\infty} \frac{\sum_{i=2}^{q-1}(1-\chi(i)) f_{i}(x)}{x^{s+1}} d x
$$

From the equation (10) we replace the $\zeta(s)$ by its $L-$ function:

$$
L(s, \chi)=\frac{1-q^{1-s}}{q} \frac{s}{(s-1)}-s \int_{1}^{+\infty} \frac{\left(1-q^{-s}\right)\{x\}-\sum_{i=2}^{q-1}(1-\chi(i)) f_{i}(x)}{x^{s+1}} d x
$$

Let's define the function $g(x)$ as follows:

$$
\begin{aligned}
g(x) & =\left(1-q^{-s}\right)\{x\}-\sum_{i=2}^{q-1}(1-\chi(i)) f_{i}(x) \\
& =\frac{1-q^{1-s}}{q}\{x\}-\left(1-\frac{1}{q}\right) r_{x}+\sum_{i=2}^{q-1}(1-\chi(i)) \sum_{k=1}^{r_{x}} \chi_{i}(k) \\
& =\frac{1-q^{1-s}}{q}\{x\}+f(x)
\end{aligned}
$$

Where $f$ is unrelated to $s$, bounded over $\mathbb{R}^{+}$and null over $[0,1]$. We can also write:

$$
\begin{aligned}
f(x) & =-\left(1-\frac{1}{q}\right) r_{x}+\sum_{i=2}^{q-1}(1-\chi(i)) \sum_{k=1}^{r_{x}} \chi_{i}(k) \\
& =\frac{r_{x}}{q}-\sum_{k=1}^{r_{x}} \chi(k)
\end{aligned}
$$

And

$$
L(s, \chi)=\left(\frac{1-q^{1-s}}{q}\right) \frac{s}{s-1}-s \int_{1}^{+\infty} \frac{\left(\frac{1-q^{1-s}}{q}\right)\{x\}+f(x)}{x^{s+1}} d x
$$

Lemma 3.5. Let's consider two variables $\sigma$ and $\tau$ such that $0<\sigma<1$, $\tau>0$ and $s=\sigma+i \tau$. Let's $q>1$ be an positive integer. Let's $L(s, \chi)$ be a L-function where $\chi$ is a Dirichlet character mod $q$ with cancellation property such that:

$$
\sum_{n=1}^{q} \chi(n)=0
$$


Let's suppose that $s=\sigma+i \tau$ is a zero for $L(s, \chi)$. i.e $L(s, \chi)=0$. Therefore

$$
\sigma=\frac{1}{2}
$$

And the Generalized Riemann's Hypothesis is true.

Proof. The proof is straightforward as we can use the lemmas 3.3 and 3.4. From lemma 3.4 we can write the following:

$$
\begin{aligned}
L(s, \chi) & =\frac{\lambda s}{s-1}-s \int_{1}^{+\infty} \frac{\lambda\{x\}+f(x)}{x^{s+1}} d x \\
\lambda & =\frac{1-q^{1-s}}{q}
\end{aligned}
$$

Where $f$ is a bounded function over $\mathbb{R}^{+}$. Then, we apply the lemma 3.3 to conclude that

$$
\sigma=\frac{1}{2}
$$

And the Generalized Riemann's Hypothesis is true.

\subsection{Conclusion}

We saw that if $s$ is a zeta zero, then real part $\Re(s)$ can only be $\frac{1}{2}$. Therefore, the Generalized Riemann Hypothesis (GRH) is true: The non-trivial zeros of a $L-$ Dirichlet function have real part equal to $\frac{1}{2}$. With this, we conclude the Factoring problem is in $\mathrm{P}$ and therefore $\mathrm{P}=\mathrm{NP}$.

\section{Acknowledgments}

I would like to thank Farhat Latrach, Giampiero Esposito, Jacques Gélinas, Michael Milgram, Léo Agélas, Ronald F. Fox, Kim Y.G, Masumi Nakajima, Maksym Radziwill and Shekhar Suman for thoughtful comments and discussions on my paper versions on the RH. All errors are mine.

\section{References}

[1] Charaf E. Chatbi A Simple Proof of the Riemann's Hypothesis https://arxiv.org/abs/1905.09385

[2] Bernhard Riemann. On the Number of Prime Numbers less than a Given Quantity https://www.claymath.org/sites/default/files/ezeta.pdf

[3] Aleksandar Ivic. The Riemann Zeta-Function: Theory and Applications 
[4] Peter Borwein, Stephen Choi, Brendan Rooney, and Andrea Weirathmueller The Riemann Hypothesis: A Resource for the Afficionado and Virtuoso Alike

http://wayback.cecm.sfu.ca/ pborwein/TEMP_PROTECTED/book.pdf

[5] Jørgen Veisdal. The Riemann Hypothesis, explained https://medium.com/cantors-paradise/the-riemann-hypothesis-explained-fa01c1f75d3f

[6] Thai Pham. Dirichlet's Theorem on Arithmetic Progressions https://web.stanford.edu/ thaipham/papers/MIT_18.104_Review_Paper.pdf

[7] G. H. Hardy. The general theory of dirichlet series. https://archive.org/details/generaltheoryofd029816mbp/page/n9

[8] Garrett, Paul. Primes in arithmetic progressions, 2011.

http : //www.math.umn.edu/ garrett/m/mfms/notes_c/dirichlet.pdf 